\title{
The Controversy Over the Use of Prozac Because of Side Effects
}

\author{
Enru Wang
}

The First High School of Changsha; Hunan; China. 410000

enruwang@sina.com

\begin{abstract}
Prozac, also known as fluoxetine, is a new class of serotonin reuptake blocker (SSRI) oral antidepressant that works by inhibiting serotonin reuptake by the central nervous system. This drug has the advantages of wide application (depression, anxiety, obsessive-compulsive disorder, bulimia, etc.) and relatively light side effects, so it has become a popular psychological drug in contemporary times. However, there are still many debates about the wide use of Prozac in the academic world. Some people are strongly opposed to the widespread use of Prozac as a psychological drug and argue that it has huge negative effects on patients. For example, Prozac can lead to more depression, increase the risk of suicide, increase anxiety, and doctors forget to tell patients that it doesn't work immediately and the side effects can be severe. After summarizing and analyzing several cases, the current conclusion of this article is that Prozac can cause some side effects, such as increased anxiety and diarrhea, but can be mitigated with positive cues and cognitive behavioral therapy.

The research methods used in the cases from references in this paper are: open field experiment, cross elevated experiment and control variable method. The authors analyzed the experimental results and tabular data to come to their own conclusions.
\end{abstract}

Keywords: Fluoxetine hydrochloride, Prozac, antidepressants, side effects, cognitive behavioral therapy, suggestion, open field trials

\section{INTRODUCTION}

The first generation of antidepressants include monoamine oxidase inhibitors (MAOI). Representative drugs include phenylethylhydrazine and moclobeamine. There are tricyclic antidepressant drugs (TCAs), representative drugs: amitriptyline, doxepin, clomipramine, drug side effects are mainly plant nervous and cardiovascular system, clinical application is reduced. The second generation of antidepressants includes tetracyclic antidepressants, which are represented by maprotiline, with similar side effects to tricyclic antidepressants. Fluoxetine is an SSRI, a new antidepressant. It has certain advantages for some patients with bulimia and is well absorbed from gastrointestinal tract after oral administration.

$\mathrm{Li}$ et al. in The Research Progress of Antidepressants, SSRI is the most widely used antidepressant in clinical practice [1]. These drugs have little effect on NE and hardly affect the recovery of DA.
In the early 1970s, based on the theory that "serotonin may be related to depression" and detailed market research, the pharmaceutical industry spent huge sums of money to develop new drugs, using the latest biochemical technology and molecular biology, "synthesis" has a selective, single "specific" action on serotonin new drug. Prozac works as well as traditional antidepressants, but with fewer side effects, breaking a three-decade rut in psychotropic drug research that began in 1950 when antidepressants were first discovered. So when Prozac was approved by the FDA in 1987, within three years, it was the best-selling antidepressant in the United States. The SAN Jose Mercury News voted Prozac as one of the greatest technological innovations of the 20th century, just as important as Freud's invention of the unconscious mind. On average, one in eight people in the United States takes Prozac [2].

However, there are still many problems to be solved in the wide application of Prozac. If you take too much of the drug, the cellular response threshold gets higher 
and higher, so that if you don't get enough of the drug, the endogenous secretion becomes even less. Although Prozac has fewer side effects than other drugs, they still baffle patients and doctors.

This article will conduct a theoretical analysis based on previous experiments, discuss the specific side effects of Prozac, judge whether the benefits of taking Prozac outweigh the risks, and how to solve these side effects. If solutions can be found, patients will have a better experience during treatment. More people with mental illness will be well off.

\section{ANALYSIS}

\subsection{Treatment principle}

Fluoxetine is a selective serotonin re-uptake inhibitor (SSRI), which can effectively inhibit the uptake of serotonin from the synaptic cleft by neurons. Serotonin is a feel-good messenger that affects almost every aspect of brain activity, from regulating mood, energy, and memory to shaping your outlook on life. Serotonin is generally regarded as a neurotransmitter, responsible for the transmission of information between neurons (Figure 1). The brain cells in the brain are directly or indirectly affected by it

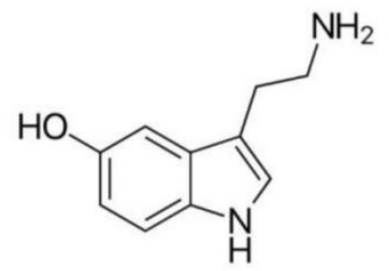

Figure 1 Molecular structural formula of the serotonin

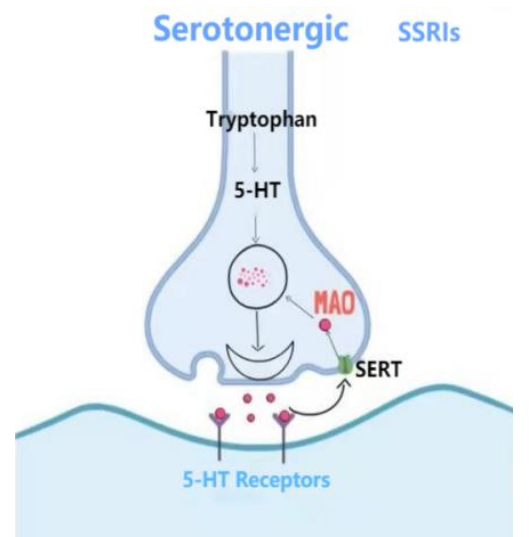

Figure 2 How serotonin works in the nervous system

High levels of serotonin are shown to enhance cognitive abilities, including memory and learning speed. And current research confirms that Serotonin can play a role in the regulation of the nervous system. As the reduction of serotonin is related to emotional depression, the mechanism of some antidepressants is to reduce the degradation of serotonin. It can temporarily increase the concentration of serotonin in the body, thereby helping to relieve emotional depression. Figure 2 shows the working principle of serotonin in the nervous system. Increasing the actual availability of this neurotransmitter in the gap can improve the emotional state and treat depressive mental disorders. Therefore, people with lower serotonin levels are more likely to experience depression, impulsive behavior, alcohol abuse, suicide, aggression, and violence[1].

\subsection{Case analysis of side effects}

\subsubsection{Case 1}

Prozac "It is just a teaser towards the mind. Citizens inside Wurtzels' book feel that the drug doesn't ease the pain and Feel to take away depression is all in the mind of a person" [2]. "The risk of suicide attempt among the patients that had taken Prozac was twice the size of those who did not take the drug [2]. Within this study, there were five suicide attempts by those who took the drug, and only one who was not administered the drug. The author insisted that while Prozac is widely regarded as a miracle fast-acting drug, for many people the drug is a death threat. It has serious side effects, leading to more depression, increased suicide attempts, increased anxiety and less hope that patients will want to live.

However, It is worth noting that the information provided in this article may not be entirely trustworthy. Most of the author's opinions are from other people's research reports, but lack evidence. In addition, the comparison experiment mentioned in this paper seems to have some shortcomings, for example, it does not take into account the consistency of other influencing factors, such as the environment around the two groups of participants.

\subsubsection{Case 2}

Another study on the association between Fluoxetine Hydrochloride and suicidal behavior

The author mentioned that, despite Martin's observation that fluoxetine was followed by negative reactions similar to those of other depression drugs, fluoxetine was found to be effective in improving the negative state of depressed patients after extensive research conducted by Fava [3]. There is no conclusive scientific evidence linking fluoxetine hydrochloride and suicidal behavior. In other words, the use of Fluoxetine Hydrochloride is not positively associated with suicidal behavior.

\subsubsection{Case 3}

The experiments concluded that short-term Fluoxetine hydrochloride administration increased anxiety-like behavior in Kunming mice through an 
open-field experiment conducted by controlling variables [4].

In this experiment, the sample Kunming mice were divided into two groups, one group was given fluoxetine hydrochloride, and the other group was given CMC solution. The mice were given continuously for 7 days.

The group of kunming mice taking fluoxetine hydrochloride (Flu, 20mg/kg) is the experimental group or Flufluoxetine hydrochloride group, and correspondingly, the group of kunming mice taking CMC solution is the control group (Figure 3). After that, open field experiment and cross elevated experiment were used Open-field experiment and cross elevated experiment are commonly used to measure the anxiety-like behavior of rodents, and measuring their probing behavior in the central area and the number and time spent in the open arm are the indicators related to anxiety. In this experiment, mice in the experimental group stayed in the central site and the open arm for longer than those in the control group, indicating that short-term administration of Fluoxetine hydrochloride (Prozac) exacerbated the anxiolytic behavior of mice.
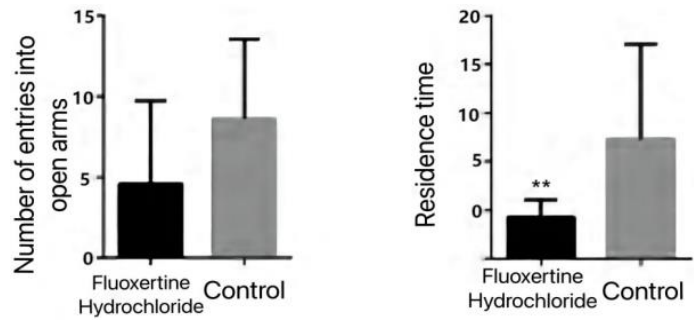

Figure 3 The charge of the situation of mice entering open arms and their staying time

\section{MITIGATION METHODS}

There is currently no clear solution to the side effects of Prozac. Aspirin is often used by people at high risk of cardiovascular disease, but gastrointestinal discomfort, bleeding and other side effects, often become resistance for some patients to adhere to medication. However, these side effects can be avoided by using enteric-soluble preparations, adding protective agents, and choosing the right time to take medication. Some studies have shown that taking Prozac in a variety of ways can reduce the side effects and harm patients.

\subsection{The combination of Fluoxetine} Hydrochloride and cognitive behavioral therapy for the treatment of depression in children and adolescents

Ma \& Guo mention that the experimenter used cognitive behavioral therapy and fluoxetine for the treatment of depression in children and adolescents[5].
Efficacy comparison Group Number of cases Recover Effective Improved Ineffective

$\begin{array}{llllll}\text { Treatment Group } & 30 & 14 & 12 & 3 & 1 \\ \text { Control Group } & 27 & 5 & 12 & 4 & 6\end{array}$

Figure 4 The comparison of curative effect between the two groups

The experimental results showed that, according to Redit, $U=3.54$. The significant efficiency of the treatment group combined with cognitive behavioral therapy was $86.67 \%$, and that of the control group was $62.96 \%$. There were 5 cases of adverse reactions in the treatment group and 20 cases of adverse reactions in the control group.

\subsection{The combination of Fluoxetine Hydrochloride and cognitive behavioral therapy in the treatment of postpartum depression}

In addition, Li \& Luo claimed that conventional Fluoxetine hydrochloride can fight postpartum depression. Postpartum depression is a common postpartum mental disorder in obstetrics and gynecology, usually occurs in the puerperium, refers to depression mood specific mainly occurs in women after childbirth.

Due to the occurrence of postpartum depression for many reasons, in part because of the influence of life events and psychological factors, so pure antidepressant treatment effect is not very ideal [6]. Studies have shown that Fluoxetine hydrochloride combined with cognitive behavioral therapy to fight postpartum depression has a very significant therapeutic effect, can effectively reduce the symptoms of postpartum depression, improve the quality of life, improve the cure rate, and high safety. Therefore, this study mainly discussed the effect of Fluoxetine hydrochloride combined with cognitive behavioral therapy in the treatment of postpartum depression.

The experimenter used the control variable method. The study samples were 60 patients with postpartum depression who received treatment in the author's hospital from January 2017 to December 2019 and were randomly divided into observation group and control group, with 30 patients in each group. In the control group, there were 18 primiparas and 12 multiparas aged 20-45 years, while in the observation group, there were 16 primiparas and 14 multiparas aged $21-44$ years. The control group received oral Fluoxetine hydrochloride alone, and the observation group received Fluoxetine hydrochloride combined with cognitive behavioral therapy. The usage and dosage of Fluoxetine hydrochloride were the same as the control group. HAMD score, clinical effect and incidence of adverse reactions were compared between the two groups before and after treatment. A lower HAMD score indicates a lower degree of depression. 


\begin{tabular}{cccc}
\hline Group & Case & Prior treatment & Posttreatmen \\
\hline Experimental Group & 30 & $28.6 \pm 6.8$ & $7.3 \pm 2.6^{\mathrm{a}}$ \\
Control Group & 30 & $28.3 \pm 7.6$ & $11.2 \pm 3.5$ \\
$t$ & & 0.161 & 4.899 \\
$P$ & & 0.872 & 0.000
\end{tabular}

Figure 5 Comparison of HAMD scores between two groups before and after treatment

The total effective rate of the observation group was $93.33 \%$. The total effective rate of the control group was $63.33 \%$. The total effective rate of the observation group was higher than the control group, and the difference was statistically significant. Comparison of the incidence of adverse reactions after treatment between the two groups The incidence of adverse reactions after treatment in the observation group was $10.00 \%(3 / 30)$, lower than $43.33 \%(13 / 30)$ in the control group, and the difference was statistically significant. Experimental results show that fluoxetine hydrochloride combined with cognitive behavioral therapy has a more significant effect than single drug therapy, effectively reducing the incidence of adverse reactions. It shows that the combination of cognitive-behavioral therapy with fluoxetine (Prozac) seems to be a good way to reduce side effects.

\subsection{Suggestion}

In addition to direct psychotherapy, there is literature suggesting that the adverse effects of Prozac use can be mitigated by hints given during medication administration. The research results showed that the clinical efficacy of patients in the experimental group was better than that in the control group, and the side effects were less than that in the control group, indicating that the suggestion of drug content could affect the efficacy and side effects of Fluoxetine [7]. It follows that doctors explain the contents of the medication and give positive cues (e.g. "You look better! "This drug seems to work particularly well for you." ) can have a significant effect not only on the therapeutic benefits of the drug but also on reducing the side effects of the drug.

\section{CONCLUSION}

When Prozac is used in clinical psychology, it does cause side effects in patients, such as the increased risk of suicide and anxiety. However, these effects can be mitigated through a variety of methods, such as positive cues and coordination with cognitive behavioral therapy. In summary, the authors prefer to combine Prozac medication with psychological counseling and positive suggestions from the physician. To be more specific, doctors can cooperate with the cognitive behavioral therapy of psychological consultants in the treatment process of various groups, and inadvertently put forward the improvement of patients' conditions during the return visit, resulting in positive psychological suggestions, so as to reduce or eliminate the side effects of Prozac in this way.

The paper still has some problems, the biggest problem is the reference sample is small, causing the conclusion is not universal. In order to solve this problem, further researches should be conducted.

In the opinion of the author, we need to pay attention to the specific combination of Prozac treatment in different environments, so that minimize the side effects of drugs and make patients get as comfortable a treatment experience as possible.

\section{ACKNOWLEDGMENT}

As a student in a traditional Chinese high school, this is my first essay. First of all, I would like to express my deepest gratitude to my high school teacher and the professor of this online course, Prof. William Trochim from Cornell University. Prof. Trochim led me to experience the university's learning mode in advance, deepened my study of psychology, and gave me precious opportunities to improve myself at this stage. Further, I would also like to thank my parents for their encouragement and support. However, as a student in a traditional high school, the real problem is how to independently search for the documents I need. Thank you very much to my classmates and friends. They did not laugh at me when I asked for help, but patiently taught me how to use various engines to find references through keywords. Also, I would like to thank my friends: the friend who encouraged me when I was not confident, and used her knowledge of biological competition to help me supplement my pharmacological knowledge. Without all their enlightening instruction and impressive kindness, I could not have completed my thesis.

\section{REFERENCES}

[1] Li M., He M., Zhang L., Qiu F., Chen W., Wu Y., Yang J., Jiang J., The Research Progress of Antidepressants, Journal of Clinical Pharmacotherapy, 14-19. doi:CNKI:SUN:LCYW.0.2017-01-002.

[2] Vares, J. The negative effect of Prozac on teenagers in the United States. (2007). https://digitalcommons.csumb.edu/cgi/viewcontent. cgi article $=1014 \&=\&$ context=caps_thes $\&=\&$ sei-re $\operatorname{dir}=1 \&$ referer

[3] Jiang H., Adverse Reactions of Fluoxetine Hydrochloride. (2008). 84-86. doi:CNKI:SUN:XDYD.0.2008-S2-041.

[4] Di T., Wang W., Niu L., Xiao R., Zhang C., Effects of short-term Fluoxetine Hydrochloride Administration on Anxiety-like Behavior in 
Kunming Mice. (2017), 144-145. doi:CNKI:SUN:BFYX.0.2017-05-117.

[5] Ma Y., Guo Y., Cognitive behavioral Therapy Combined with Fluoxetine in the Treatment of Depression in Children and Adolescents, Chinese Disability Medicine. (2015), 160.

[6] Li D., Luo Z., Fluoxetine Hydrochloride Combined with Cognitive Behavioral Therapy in the Treatment of Postpartum Depression, doi : 10.14164/j.cnki.cn11-5581/r.2021.17.094.

[7] Chen X., Zhang C., Effect of Suggestion on the Efficacy and Side Effects of Fluoxetine, (2003), 9-10.doi:CNKI:SUN:QLHL.0.2003-07-005. 